\title{
Roles of d- and f-orbital electrons in the complexation of Eu(III) and Am(III) ions with alkyldithiophosphinic acid and alkylphosphinic acid using scalar-relativistic DFT calculations
}

\author{
Masashi Kanekoa, Masayuki Watanabe ${ }^{a, *}$, Sunao Miyashita ${ }^{b}$, Satoru Nakashima ${ }^{b, c}$ \\ ${ }^{a}$ Nuclear Science and Engineering Center, Japan Atomic Energy Agency, 2-4, Shirakata, Tokai-mura, Naka-gun, \\ Ibaraki-ken 319-1195, Japan \\ ${ }^{\mathrm{b}}$ Graduate School of Science, Hiroshima University, 1-3-1, Kagamiyama, Higashi-Hiroshima, Hiroshima-ken 739- \\ 8526, Japan \\ 'Natural Science Center for Basic Research and Development, Hiroshima University, 1-4-2, Kagamiyama, \\ Higashi-Hiroshima, Hiroshima-ken 739-8526, Japan
}

Received November 14, 2016; Accepted March 3, 2017; Published online March 21, 2017

Scalar-relativistic density functional calculations were applied to the complexation of Eu(III) and Am(III) ions with alkyldithiophosphinic acid (S-donor) and alkylphosphinic acid (O-donor) from the viewpoint of the bonding nature of valence orbitals of a metal ion. Two and four conformers for the S-donor and O-donor complexes, respectively, were optimized at the ZORA-BP86 level. The stabilization energies by complex formation between the metal ions and the ligands toward the hydrated metal ions were estimated at the ZORA-B2PLYP level; these calculations reproduced the experimental $\mathrm{Am} / \mathrm{Eu}$ selectivities, where the S-donor ligand preferably coordinates to $\mathrm{Am}(\mathrm{III})$ ion rather than $\mathrm{Eu}(\mathrm{III})$ ion, whereas O-donor ligand selectively coordinates to $\mathrm{Eu}(\mathrm{III})$ ion rather than Am(III) ion. Electron population analyses based on the molecular orbitals indicated that the d-orbital electrons in both the Eu and Am complexes participate in bonding covalent interactions with donor atoms and have surprisingly similar contributions. This result suggests that the covalent interaction between the d-orbital electrons in the metal ion and the ligands may be due to the geometrical similarity of the molecular structures of the Eu and Am complexes. In contrast, the contributions of the f-orbital electrons in the metal ions were different in the Eu and Am complexes. In the case of the S-donor complexes, non-bonding and bonding contributions were observed for the $\mathrm{Eu}$ and $\mathrm{Am}$ complexes, respectively, and in the case of the O-donor complex, bonding and anti-bonding contributions were observed for the Eu and Am complexes, respectively. This result implies that the selectivities of $\mathrm{Eu}(\mathrm{III})$ and $\mathrm{Am}(\mathrm{III})$ ions were determined by the difference in the participation of their f-orbital electrons, not their d-orbital electrons, in covalent interactions.

\section{Introduction}

High-level radioactive liquid waste (HLLW) containing many types of metal ions is generated after the reprocessing of spent nuclear fuel. Minor-actinides (MA: Am and $\mathrm{Cm}$ ) have long-term radiotoxicity due to their long half-lives and $\alpha$-activities; thus, the partitioning and transmutation is a rational method for their disposal. In this method, MA ions are separated from HLLW, followed by transmutation of the MA ions to short-lived nuclides; this method was developed in order to reduce their environmental loads. ${ }^{1}$ However, the selective separation of MA ions from lanthanide ( $\mathrm{Ln}$ ) ions is difficult due to the similarity of their chemical properties, such as their oxidation states, geometric structures, and chemical stabilities in solution. ${ }^{2}$ To effectively separate MA ions, the molecular design of separation materials that have high selectivity for MA ions over $\mathrm{Ln}$ ions is desirable.

Solvent extraction has been employed for the separation of MA ions from Ln ions. ${ }^{3-5}$ Selectivities for MA and Ln ions have been investigated using various extraction reagents. Previous studies indicated that $\mathrm{S}$ - and $\mathrm{N}$-donor ligands such as alkyldithiophosphinic acid ${ }^{6,7}\left(\mathrm{R}_{2} \mathrm{PS}_{2} \mathrm{H}\right.$; Figure 1a) and $N, N, N^{\prime}, N^{\prime}$-tetrakis(4-pyridylmethyl)ethylenediamine ${ }^{8}$ (TPEN), respectively, preferably coordinate to MA ions over Ln ions, whereas $\mathrm{O}$-donor ligands such as alkylphosphinic acid ${ }^{6}$

*Corresponding author. E-mail: watanabe.masayuki@jaea.go.jp
$\left(\mathrm{R}_{2} \mathrm{PO}_{2} \mathrm{H}\right.$; Figure 1 b) selectively coordinate to $\mathrm{Ln}$ ions over MA ions. This dependency of MA/Ln selectivity on donor atoms has been explained using Pearson's hard and soft acids and bases (HSAB) rule. ${ }^{9}$ S- and N-donor atoms are softer bases than $\mathrm{O}$-donor atoms; they bond more strongly to MA ions than Ln ions because MA ions are softer acids than Ln ions due to the extended distribution of their electron orbitals in the valence region. ${ }^{10}$ The difference in covalency between MA and Ln complexes may determine the separation behaviors of MA ions from Ln ions; however, it is difficult to quantitatively discuss covalency using the HSAB rule.

Density functional theory (DFT) is a powerful tool to understand the chemical stabilities and electronic states of f-block compounds, ${ }^{11-14}$ which has been successfully applied to evaluation of feasibility to separate MA ions from Ln ions. ${ }^{15}$ Discussion of the nature of the bonding between the metal ion and ligands in Ln and MA complexes has been controversial.<smiles>[R]P([R])(=S)S</smiles><smiles>[R]P([R])(=O)O</smiles>

(a) alkyldithiophosphinic acid

(b) alkylphosphinic acid

Figure 1. Molecular structures of (a) S- and (b) O-donor extraction ligands. 
Cao et al. ${ }^{16}$ applied f-in-core relativistic effective core potential (RECP), which does not consider f-orbital electrons explicitly but sets that their contributions using a projector operator, to MA/Ln separation by alkyldithiophosphinic acid ${ }^{17}$ and alkylphosphinic acid. ${ }^{18}$ They suggested that the selectivity of MA and Ln ions is determined by the contribution of s-, p-, and d-orbital electrons, not f-orbital electrons, in the metal ion. ${ }^{17}$ Meanwhile, our previous study using the segmented all-electron relativistic contracted (SARC) basis set, ${ }^{19-21}$ which explicitly includes f-orbital electrons, indicated that the difference in the contribution of f-orbital electrons to metal-ligand bonding between $\mathrm{Eu}(\mathrm{III})$ and $\mathrm{Am}(\mathrm{III})$ ions contributes to the $\mathrm{Am} / \mathrm{Eu}$ selectivity. ${ }^{22}$

This study elucidates the role of the atomic orbitals of the metal ions in the complexation behavior of Eu(III) and Am(III) ions with alkyldithiophosphinic acid and alkylphosphinic acid using DFT calculations. It focuses on the relative stability of the metal ion by complexation reaction and discusses the covalent interactions of the atomic orbitals in the metal with the donor atoms of the ligands. More specifically, the d-and f-orbital electrons of the metals and their major contributions to the electron populations were intensively studied here. We aim to investigate the correlation between the covalency of f-block compounds and the separation mechanism of MA ions from Ln ions. We also suggest an answer to the question of the origin of the selectivity of MA and Ln ions.

\section{Calculations}

In this study, we ignored the detailed reaction mechanism, such as subsequently complex formation and dehydration processes, in order to simply correlate Am/Eu selectivity with relative stability between initial and final states. This assumption is crude when estimating the absolute value of Gibbs free energy difference $(\Delta G)$, because the effect of solvation and dehydration entropy are not included. It is, however, valid for the comparison of the Gibbs free energy difference between Eu and Am systems, because their coordination geometries are almost the same and the effect of stepwise dehydration or formation is finally cancelled when estimating the difference of $\Delta G$ between Eu and Am systems. We considered the stabilization of hydrated $\mathrm{Eu}$ (III) and Am(III) ions by the formation of complexes and modeled the complexation reactions, as illustrated in Figure 2. Solvent extraction experiments have reported that one metal ion of $\mathrm{Ln}$ or MA forms a complex with three equivalents of monomeric $\mathrm{R}_{2} \mathrm{PS}_{2} \mathrm{H}(\mathrm{R}=$ 2,4,4-trimethylpentyl) and dimeric $\mathrm{R}_{2} \mathrm{PO}_{2} \mathrm{H}(\mathrm{R}=2,4,4$-trimethylpentyl) ligands. ${ }^{23}$ The coordination geometries of these complexes in solution, investigated by extended X-ray absorption fine structure (EXAFS) experiment, are suggested to be
$\mathrm{MS}_{6}$ and $\mathrm{MO}_{6}(\mathrm{M}=\mathrm{Sm}$ and $\mathrm{Cm})$ environments for alkyldithiophosphinic and alkylphosphinic acids, respectively. ${ }^{23}$ The geometrical coordinates of the metal complexes were created by referring to the single crystal X-ray diffraction data of the nona-hydrated complexes, $\left[\mathrm{M}\left(\mathrm{H}_{2} \mathrm{O}\right)_{9}\right]^{3+}(\mathrm{M}=\mathrm{Eu}$ and $\mathrm{Am}),{ }^{24,25}$ and the S-donor complex, $\left[\mathrm{Sm}\left(\mathrm{R}_{2} \mathrm{PS}_{2}\right)_{3}\right](\mathrm{R}=\text { cyclohexyl })^{26}$. However, this could not be performed for the O-donor complexes, $\left[\mathrm{M}\left\{\left(\mathrm{R}_{2} \mathrm{PO}_{2}\right)_{2} \mathrm{H}\right\}_{3}\right]$, because their crystal structures are not available.

Here, we considered simple models for both the S-donor and O-donor complexes in which all the alkyl chains of the phosphine group were replaced with methyl substituents. We believe that the MA/Ln selectivity is primarily determined by the coordination bond between the metal ion and the ligands, not by the hydrophobic properties of the alkyl chains. This is because the length of the alkyl chain of an extraction ligand affects its performance for extracting the metal ions but minimally affects its selectivity for MA/Ln ions, as indicated by some studies. ${ }^{27,28}$ The stereoisomerism of f-block complexes influences their spectroscopic properties ${ }^{29}$ and their Am/Eu selectivities. ${ }^{30}$ Some stereoisomers exist for the S-donor and $\mathrm{O}$-donor complexes based on the chelating effects of the ligands (Figure 3). The S-donor complex, $\left[\mathrm{M}\left(\mathrm{Me}_{2} \mathrm{PS}_{2}\right)_{3}\right]$, has two geometrical isomers $(\Delta, \Lambda)$ that are crystallographically inequivalent, as observed in single crystal X-ray diffraction experiments (Figure 3a). ${ }^{26}$ In the case of the O-donor complex, $\left[\mathrm{M}\left\{\left(\mathrm{Me}_{2} \mathrm{PO}_{2}\right)_{2} \mathrm{H}\right\}_{3}\right]$, we considered four conformers $(\Delta[\delta \delta \delta], \Delta[\lambda \lambda \lambda], \Lambda[\delta \delta \delta]$, and $\Lambda[\lambda \lambda \lambda])$ based on the screwing direction of the ligands toward the plane comprising a metal ion and bidentate $\mathrm{O}$-donor atoms, denoted as $\delta$ or $\lambda$, as well as the $\Delta$ and $\Lambda$ isomers (Figure $3 \mathrm{~b}$ ). In this case, assuming that the $\mathrm{O}$-donor complex has pseudo $C_{3}$ symmetry as observed in the case of the S-donor complex, ${ }^{26}$ we did not consider the $\delta \delta \lambda$ and $\lambda \lambda \delta$ conformers.

All DFT calculations were performed by the quantum chemical calculation package ORCA ver. 3.0 developed by Neese et al. ${ }^{31}$ The scalar-relativistic effect was considered by the zeroth-order regular approximation (ZORA) Hamiltonian ${ }^{32}$ using the formalism implemented by Wüllen. ${ }^{33}$ The spin-orbit coupling effect was included in the present calculations using the Breit-Pauli perturbation method. ${ }^{34}$ The SARC basis set, optimized for scalar-relativistic ZORA calculations, was assigned to all atoms. ${ }^{19-21}$ The spin multiplicities of the $\mathrm{Am}(\mathrm{III})$ and $\mathrm{Eu}(\mathrm{III})$ complexes were set to the spin septet state. The unrestricted Kohn-Sham method was applied to the open-shell system. BP86 $6^{35,36}$ and B2PLYP ${ }^{37}$ exchange-correlation functionals were employed for geometry optimization and single-point calculations, respectively, under gas conditions because these methods have been confirmed in our previous studies to reproduce the equilibrium structures, the strengths

(a) S-donor

$$
\mathrm{M}^{\prime \prime \prime}\left(\mathrm{H}_{2} \mathrm{O}\right)_{9}{ }^{3+}+3 \mathrm{~S}_{\mathrm{S}}^{\mathrm{Me}}
$$<smiles>CSP1SP2SC3(S1)S[PH](C)(S2)S3</smiles>

(b) O-donor

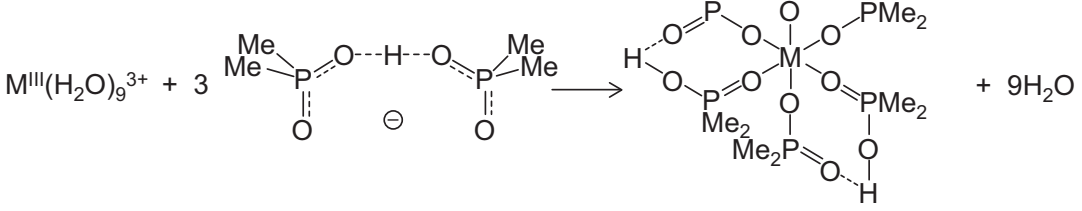

Figure 2. Reaction models of the complexation of Eu(III) or Am(III) ion with (a) S- and (b) O-donor ligands in aqueous solution. 
(a) S-donor complex $\left[\mathrm{M}\left(\mathrm{Me}_{2} \mathrm{PS}_{2}\right)_{3}\right]$

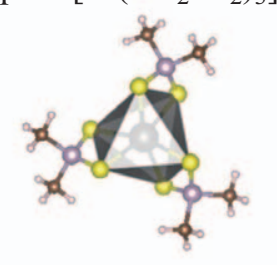

$\triangle$

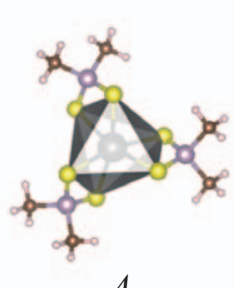

$\Lambda$

(b) O-donor complex $\left[\mathrm{M}\left\{\left(\mathrm{Me}_{2} \mathrm{PO}_{2}\right)_{2} \mathrm{H}\right\}_{3}\right]$

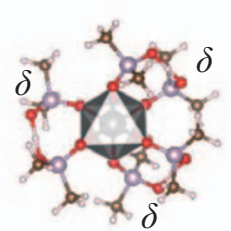

$\Delta[\delta \delta \delta]$

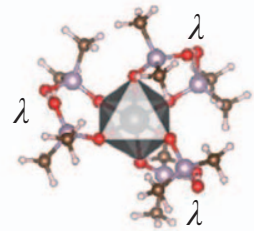

$\Delta[\lambda \lambda \lambda]$

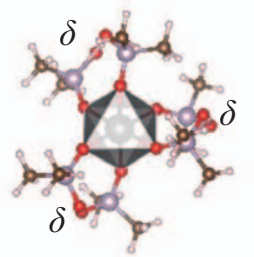

$\Delta[\delta \delta \delta]$

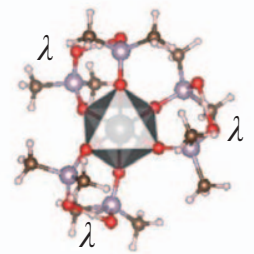

$\Delta[\lambda \lambda \lambda]$

Figure 3. Three-dimensional descriptions of the conformers of (a) S- and (b) O-donor complexes. The black, purple, yellow, red, brown, and pink spheres represent metal, phosphorus, sulfur, oxygen, carbon, and hydrogen atoms, respectively.

of coordination bonds and the relative stabilities of complexation reactions of f-block compounds. ${ }^{22}$ Equilibrium structure searches were performed without any geometrical constraints, followed by normal vibrational frequency calculations. Resolution of the identity (RI) approximations, Split-RI-J ${ }^{38}$ and RIJCOSX, ${ }^{39}$ were employed for all self-consistent field (SCF) calculations for the pure-DFT and hybrid-DFT computations, respectively. For the detailed computational conditions, such as the accuracy of self-consistent field calculations and the level of basis sets, please refer to Reference 22 .

\section{Results and discussion}

3.1. Comparison of stereoisomer. Normal vibrational frequency analysis after geometry optimization indicated that all the obtained molecular structures were located in local minima. The three-dimensional geometries of the optimized structures of the S- and O-donor complexes were visualized using VESTA 3.0 program $^{40}$ and are shown in Figure 3. All the S-donor complexes have distorted octahedral geometries, whereas the $\mathrm{MO}_{6}$ arrangement of the $\mathrm{O}$-donor complexes belongs to the pseudo $O_{\mathrm{h}}$ point group. These results were due to the difference in the bite angles between the monomeric S-donor and dimeric O-donor ligands. ${ }^{17,18,23}$ Table 1 shows a comparison of the metal-ligand distances of the Eu and Am complexes with S- and O-donor ligands. In the case of the $\mathrm{S}$-donor complexes, the distances between the metal ion and the $\mathrm{S}$ atoms and between the metal ion and the $\mathrm{P}$ atoms were ca. 2.84 and $3.39-3.40 \AA$, respectively. In the case of the O-donor complexes, both the Eu and Am complexes have similar geometries wherein the metal-ligand distances were 2.31 $2.36 \AA$ and $3.66-3.76 \AA$ for M-O and M-P, respectively. The variation of bond length among stereoisomers was small for both $\mathrm{S}$-donor and $\mathrm{O}$-donor complexes. These bond lengths were consistent with the experimental values for $\mathrm{Sm}$ and $\mathrm{Cm}$ complexes. $^{23}$

Gibbs free energy difference $(\Delta G)$ for the complexation reaction was calculated as the energy difference in the sum of Gibbs free energy values of the reactants and products, as described in Figure 2. The Gibbs free energy $(G)$ was divided into total energy $\left(E^{\text {tot }}\right)$ obtained by single-point calculations at the B2PLYP level and a thermal correction term of Gibbs free energy $\left(G^{\text {corr }}\right)$ obtained by calculating the normal vibration modes at the BP86 level (eq 1). The $G^{\text {corr }}$ term includes a thermal correction for the enthalpy $\left(H^{\text {corr }}\right)$, the entropy term $(S)$,
TABLE 1: Calculated metal-ligand distances for $\mathrm{Eu}$ and Am complexes with $\mathrm{S}$ - and $\mathrm{O}$-donor ligands

\begin{tabular}{ccccc}
\hline \multirow{2}{*}{ Complex } & \multicolumn{2}{c}{$\mathrm{M}-\mathrm{S}_{\text {ave }}$ or $\mathrm{M}-\mathrm{O}_{\text {ave }} / \AA$} & \multicolumn{2}{c}{$\mathrm{M}-\mathrm{P}_{\text {ave }} / \AA$} \\
\cline { 2 - 5 } & $\mathrm{M}=\mathrm{Eu}$ & $\mathrm{M}=\mathrm{Am}$ & $\mathrm{M}=\mathrm{Eu}$ & $\mathrm{M}=\mathrm{Am}$ \\
\hline $\mathrm{M}\left(\mathrm{Me}_{2} \mathrm{PS}_{2}\right)_{3}$ & & & & \\
$\Delta$ & 2.844 & 2.839 & 3.398 & 3.389 \\
$\Lambda$ & 2.844 & 2.839 & 3.399 & 3.389 \\
& 2.803 & 2.826 & 3.40 & 3.45 \\
Exp. $^{a}$ & $(M=S m)$ & $(M=C m)$ & $(M=S m)$ & $(M=C m)$ \\
\hline $\mathrm{M}\left\{\left(\mathrm{Me}_{2} \mathrm{PO}_{2}\right)_{2} \mathrm{H}\right\}_{3}$ & & & & \\
$\Delta[\delta \delta \delta]$ & 2.312 & 2.355 & 3.711 & 3.730 \\
$\Delta[\lambda \lambda \lambda]$ & 2.319 & 2.357 & 3.663 & 3.689 \\
$\Lambda[\delta \delta \delta]$ & 2.319 & 2.354 & 3.685 & 3.748 \\
$\Lambda[\lambda \lambda \lambda]$ & 2.311 & 2.352 & 3.718 & 3.764 \\
Exp. $^{a}$ & 2.301 & 2.32 & 3.83 & - \\
\hline & $(M=S m)$ & $(M=C m)$ & $(M=S m)$ & - \\
\hline
\end{tabular}

${ }^{a}$ Reference 23.

and temperature (T), as shown in eq 2. The $H^{\text {corr }}$ and $S$ terms include contributions from vibration (vib), which is based on the approximation of a strictly harmonic oscillator, rotation (rot). The rotation here is assumed as a rigid rotor, and translation (trans) (eqs 3 and 4$). S^{\text {spin }}$ denotes the entropy term generated from the spin multiplicity of compound. All $G^{\text {corr }}$ values were calculated under standard state condition $(298.15 \mathrm{~K}, 1$ atom).

$$
\begin{aligned}
& G=E^{\text {tot }}+G^{\text {corr }} \\
& G^{\text {corr }}=H^{\text {corr }}-T S \\
& H^{\text {corr }}=H^{\text {trans }}+H^{\text {vib }}+H^{\text {rot }} \\
& S=S^{\text {trans }}+S^{\text {vib }}+S^{\text {rot }}+S^{\text {spin }}
\end{aligned}
$$

Table 2 shows the $\Delta E^{\text {tot }}(\mathrm{M}), \Delta G^{\text {corr }}(\mathrm{M})$, and $\Delta G(\mathrm{M})$ values for the $\mathrm{S}$ - and $\mathrm{O}$-donor complexes. In the case of the $\mathrm{S}$-donor complexes, the $\Delta G(\mathrm{Am})$ values are more negative than the $\Delta G(\mathrm{Eu})$ values for both $\Delta$ and $\Lambda$ conformers. In the case of the $\mathrm{O}$-donor system, the $\Delta G(\mathrm{Eu})$ values are smaller than the $\Delta G(\mathrm{Am})$ values for the $\Delta[\delta \delta \delta], \Delta[\lambda \lambda \lambda], \Lambda[\delta \delta \delta]$, and $\Lambda[\lambda \lambda \lambda]$ conformers. Defining $\Delta \Delta G$ as $\Delta G(\mathrm{Eu})-\Delta G(\mathrm{Am})$, none of the 
TABLE 2: $\Delta E^{\text {tot }}(\mathrm{M}), \Delta G^{\text {corr }}(\mathrm{M})$, and $\Delta G(\mathrm{M})$ for $\mathrm{M}=\mathrm{Eu}$ and $\mathrm{Am}$ complexes with $\mathrm{S}$ - and $\mathrm{O}$-donor ligands and their average values with standard deviations

\begin{tabular}{ccccccc}
\hline \multirow{2}{*}{ Complex } & \multicolumn{2}{c}{$\Delta E^{\text {tot }}(\mathrm{M}) / \mathrm{kJ} \mathrm{mol}^{-1}$} & \multicolumn{2}{c}{$\Delta G^{\mathrm{corr}}(\mathrm{M}) / \mathrm{kJ} \mathrm{mol}^{-1}$} & \multicolumn{2}{c}{$\Delta G(\mathrm{M}) / \mathrm{kJ} \mathrm{mol}^{-1}$} \\
\cline { 2 - 7 } $\mathrm{M}=\mathrm{Eu}$ & & $\mathrm{M}=\mathrm{Am}$ & $\mathrm{M}=\mathrm{Eu}$ & $\mathrm{M}=\mathrm{Am}$ & $\mathrm{M}=\mathrm{Eu}$ & $\mathrm{M}=\mathrm{Am}$ \\
\hline $\mathrm{M}\left(\mathrm{Me}_{2} \mathrm{PS}_{2}\right)_{3}$ & -1928.42 & -1947.52 & -249.06 & -247.39 & -2177.48 & -2194.92 \\
$\Delta$ & -1928.98 & -1945.78 & -250.44 & -253.25 & -2179.42 & -2199.03 \\
$\Lambda$ & -1928.70 & -1946.65 & -249.75 & -250.32 & -2178.45 & -2196.98 \\
Average & 0.28 & 0.87 & 0.69 & 2.93 & 0.97 & 2.06 \\
Std. dev. & & & & -2400.55 & -2380.51 \\
$\mathrm{M}\left\{\left(\mathrm{Me}_{2} \mathrm{PO}_{2}\right)_{2} \mathrm{H}\right\}_{3}$ & -2138.83 & -2122.46 & -261.72 & -258.06 & -2393.16 & -2374.12 \\
$\Delta[\delta \delta \delta]$ & -2135.75 & -2114.56 & -257.40 & -259.56 & -236 & -2371.42 \\
$\Delta[\lambda \lambda \lambda]$ & -2134.49 & -2116.22 & -260.99 & -255.20 & -2395.48 & -2385.57 \\
$\Lambda[\delta \delta \delta]$ & -2141.88 & -2125.37 & -256.12 & -260.21 & -2398.00 & -2377.91 \\
$\Lambda[\lambda \lambda \lambda]$ & -2137.74 & -2119.65 & -259.06 & -258.25 & -2396.80 & 5.52 \\
Average & 2.86 & 4.42 & 2.36 & 1.93 & 2.76 & \\
Std. dev. & & & & & & \\
\hline
\end{tabular}

$\Delta \Delta G$ were dependent on the conformations of the S-donor and O-donor complexes. The average $\Delta \Delta G$ values were $18.5 \pm 1.1$ $\mathrm{kJ} \mathrm{mol}^{-1}$ for the $\mathrm{S}$-donor system and $-18.9 \pm 4.2 \mathrm{~kJ} \mathrm{~mol}^{-1}$ for the $\mathrm{O}$-donor system. These results reproduced the experimental selectivities and relative stabilities of Am(III) and $\mathrm{Eu}(\mathrm{III})$ ions; bis-(2,4,4-trimethylpentyl)-dithiophosphinic acid and bis-(2,4,4-trimethylpentyl)phosphinic acid showed separation factors $\left(D_{\mathrm{Am}} / D_{\mathrm{Eu}}\right)$ of $5900^{7}$ and $0.058,{ }^{6}$ respectively, corresponding to $\Delta \Delta G=21.5$ and $-7.1 \mathrm{~kJ} \mathrm{~mol}^{-1}$, respectively. Furthermore, the difference of the $\Delta G^{\text {corr }}(\mathrm{M})$ values between the Eu and Am systems was smaller than that of the $\Delta E^{\text {tot }}(\mathrm{M})$ values. For example, in the case of the O-donor complex, the average $\Delta G^{\text {corr }}(\mathrm{M})$ values were $-259.1 \pm 2.4$ and $-258.3 \pm 1.9$ $\mathrm{kJ} \mathrm{mol}^{-1}$ for $\mathrm{M}=\mathrm{Eu}$ and $\mathrm{Am}$, respectively. In contrast, the average $\Delta E^{\text {tot }}(\mathrm{M})$ values were $-2396.8 \pm 2.8$ and $-2377.9 \pm 5.5$ $\mathrm{kJ} \mathrm{mol}^{-1}$ for $\mathrm{M}=\mathrm{Eu}$ and Am, respectively. This indicates that the dominant contribution to the estimation of $\Delta G(\mathrm{M})$ is $\Delta E^{\mathrm{tot}}(\mathrm{M})$ which based on the orbital interactions, and not $\Delta G^{\mathrm{corr}}(\mathrm{M})$. In other words, the $\mathrm{Am} / \mathrm{Eu}$ selectivity in this case should be determined by the difference between the orbital interactions of the Eu and Am complexes, i.e., the chemical bonding.

3.2. Orbital interactions by population analysis. To discuss the orbital interactions in Eu and Am complexes with Sand $\mathrm{O}$-donor ligands, the atomic spin population of the metal ions, $\rho_{\text {spin }}(\mathrm{M})$, was estimated using Mulliken's procedure (Table 3). ${ }^{41}$ The absolute values of $\rho_{\text {spin }}(\mathrm{M})$ of the S-donor complex were greater than those of the $\mathrm{O}$-donor complex for both the Eu and Am systems. This may be explained by the fact that the decomposition of the electronic degeneracy of orbital levels caused by the decrease of the octahedral symmetry of the S-donor complexes, which belong to the pseudo- $D_{3}$ point group, resulted in an increase of the orbital overlap between the center metal and donor atoms. In all the cases, the f-orbital electrons made a dominant contribution to $\rho_{\text {spin }}(\mathrm{M})$. Comparing the atomic orbital components of $\rho_{\text {spin }}(\mathrm{M})$ between the Eu and Am complexes, the contributions of the dand f-orbital electrons of the Am complexes were greater than those of the Eu complexes, whereas the contributions of the sand $\mathrm{p}$-orbital electrons to $\rho_{\text {spin }}(\mathrm{M})$ did not vary significantly between the Eu and Am complexes for both the S- and $\mathrm{O}$-donor systems. This indicates that the difference in the contributions of d- and f-type atomic orbital electrons of $\mathrm{Eu}(\mathrm{III})$ and Am(III) ions to their complexes may correlate with $\mathrm{Am} / \mathrm{Eu}$ selectivity. Figure 4 shows selected MO surface
TABLE 3: Mulliken's atomic spin population of Eu and Am ions for S- and O-donor complexes

\begin{tabular}{cccccc}
\hline \multirow{2}{*}{ Complex } & \multicolumn{5}{c}{$\rho_{\text {spin }}(\mathrm{M}) /$ electrons } \\
\cline { 2 - 6 } & all & $\mathrm{s}$ & $\mathrm{p}$ & $\mathrm{d}$ & $\mathrm{f}$ \\
\hline $\mathrm{M}\left(\mathrm{Me}_{2} \mathrm{PS}_{2}\right)_{3}(\Delta)$ & & & & & \\
$\mathrm{M}=\mathrm{Eu}$ & 6.129 & 0.015 & 0.027 & 0.097 & 5.990 \\
$\mathrm{M}=\mathrm{Am}$ & 6.138 & 0.024 & 0.033 & 0.134 & 5.948 \\
\hline $\mathrm{M}\left\{\left(\mathrm{Me}_{2} \mathrm{PO}_{2}\right)_{2} \mathrm{H}\right\}_{3}(\Delta[\delta \delta \delta])$ & & & & & \\
$\mathrm{M}=\mathrm{Eu}$ & 6.038 & 0.006 & 0.016 & 0.047 & 5.969 \\
$\mathrm{M}=\mathrm{Am}$ & 6.020 & 0.011 & 0.015 & 0.068 & 5.926 \\
\hline
\end{tabular}

(a) d-type MOs

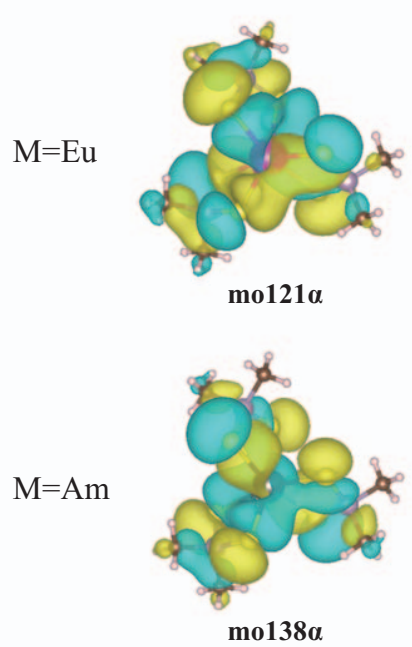

(b) f-type MOs
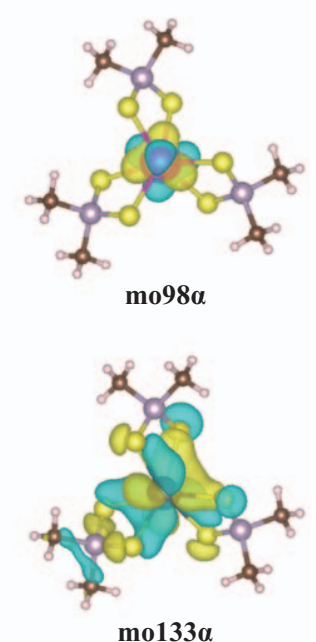

Figure 4. Selected MO surface descriptions of (a) d-type and (b) f-type MOs for S-donor complexes were visualized at $2.5 \times 10^{-5}$ electrons/bohr ${ }^{3}$.

descriptions for S-donor complexes. In the case of d-type MOs, the bond overlap between metal ion and donor atoms was indicated to be same for Eu and Am systems. In the case of f-type MOs, there was no overlap between $\mathrm{Eu}^{3+}$ ion and donor atoms, whereas the explicit orbital interaction between $\mathrm{Am}^{3+}$ ion and donor atoms was observed. This result suggested that the orbital overlap between metal ion and donor 
(a) S-donor

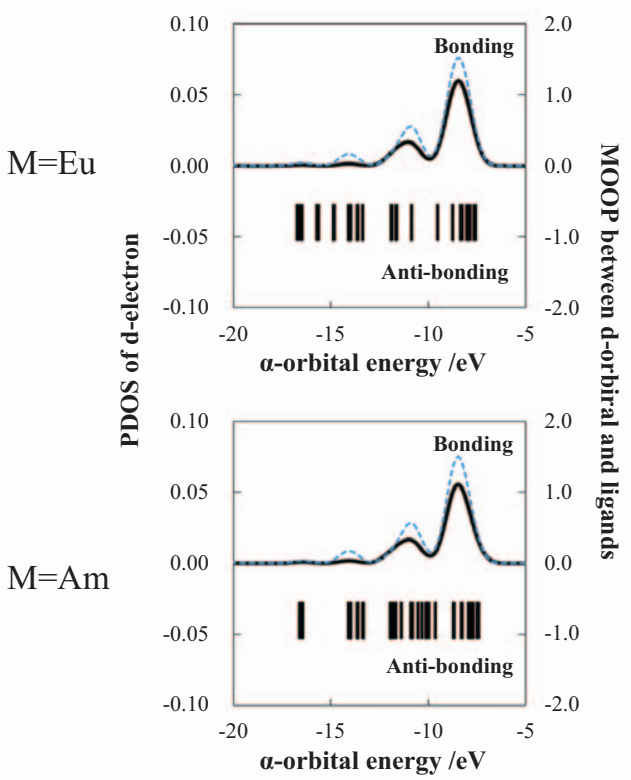

(b) O-donor

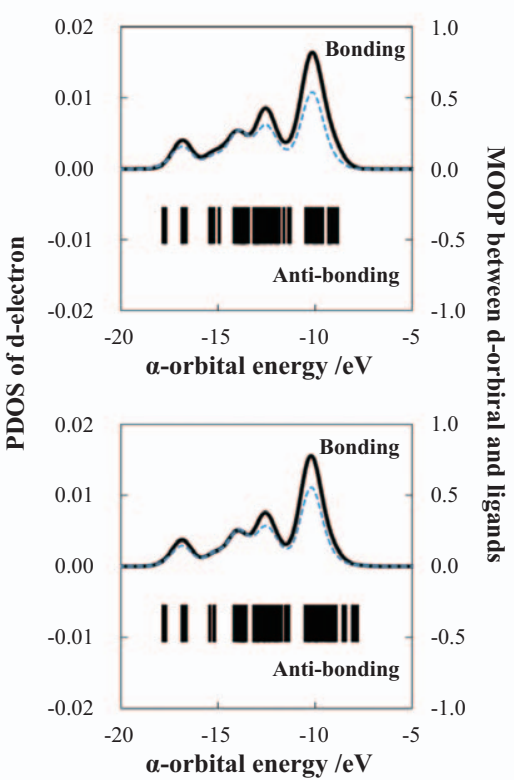

Figure 5. Partial density of states (PDOS) curves of the d-orbital electrons of metal ions (black solid line) and MO overlap population (MOOP) curves between the d-orbital of the metal ions and the donor-atoms of the ligands (blue broken line) with orbital energy level (black bar) in the valence $\alpha$-spin MO region for Eu and Am complexes with S- and O-donor ligands.

(a) S-donor

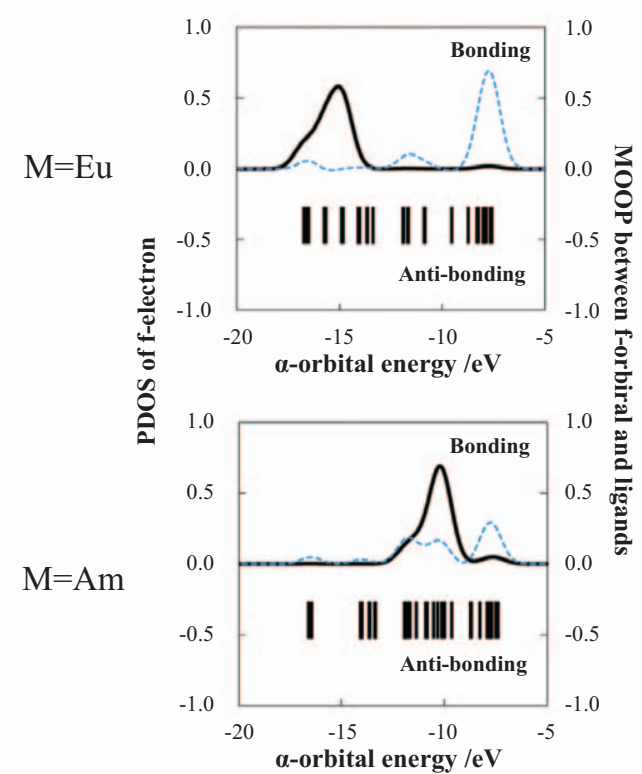

(b) O-donor

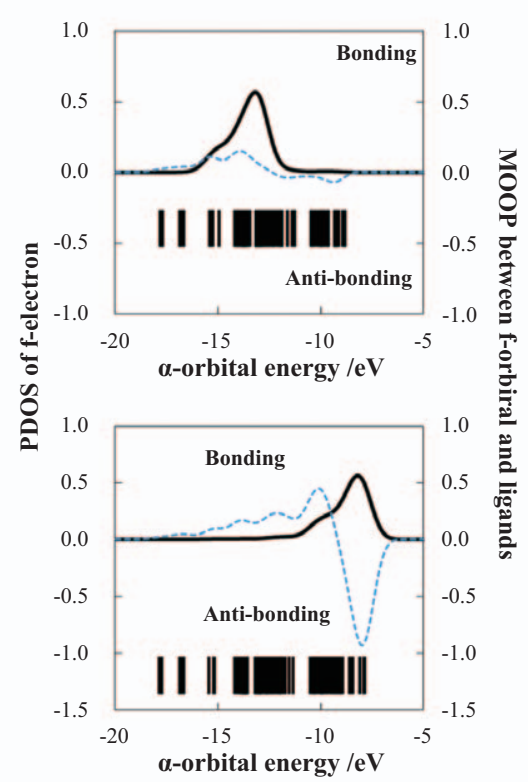

Figure 6. Partial density of states (PDOS) curves of the f-orbital electrons of metal ions (black solid line) and MO overlap population (MOOP) curves between the f-orbital of the metal ions and the donor-atoms of the ligands (blue broken line) with orbital energy level (black bar) in the valence $\alpha$-spin MO region for Eu and Am complexes with S- and O-donor ligands.

atoms was a critical factor to understand the Am/Eu selectivity.

Molecular orbital overlap population (MOOP), which enables us to evaluate the strength and sign of the bonding overlap between AOs in each MO, was applied to these complexes. ${ }^{42}$ Recently, this analysis was employed in computational studies of the bonding properties of f-block compounds. ${ }^{43-45}$ The MOOP of the $i$ th MO, termed $O P^{i}$, can be described in terms of eq 5 :

$$
O P^{i}=2 \Sigma_{\mu} \Sigma_{v} c_{\mu}{ }^{i} c_{v}{ }^{i} S_{\mu v}
$$

where $c_{\mu}$ and $c_{v}$ denote the MO coefficients of the basis func- tions $\chi_{\mu}$ and $\chi_{v}$, respectively, and $S_{\mu v}$ denotes the overlap integral between $\chi_{\mu}$ and $\chi_{v}$. To discuss the property of the bonding between the d- or f-orbital of the metal ion and the donor atoms, $\mu$ was defined as belonging to the set of the d- or f-type basis functions in the metal ion, and $v$ was defined as belonging to the set of all basis functions in the donor atoms. The partial density of states (PDOS) curves of the AOs in the metal ion, with MOOP between the AOs in the metal ion and the donor atoms in the valence $\alpha$-spin orbital region and with selected MO surfaces, are shown in Figures 5 and 6. These curves can be described as a Gaussian line shape profile convoluted with a half-width of $0.5 \mathrm{eV}$ using the single-point calculation results of the $\Delta$ conformer of $\left[\mathrm{M}\left(\mathrm{Me}_{2} \mathrm{PS}_{2}\right)_{3}\right]$ and the 
$\Delta[\delta \delta \delta]$ conformer of $\left[\mathrm{M}\left\{\left(\mathrm{Me}_{2} \mathrm{PO}_{2}\right)_{2} \mathrm{H}\right\}_{3}\right]$.

The MOOP curve between the d-orbital in the metal ion and the donor atoms correlates to the PDOS of the d-orbital in the metal ion with a positive linearity in all systems (Figure 5), indicating that the d-orbital in either the Eu(III) ion or Am(III) ion participates in a bonding covalent interaction with the Sand $\mathrm{O}$-donor atoms, as observed in the MO surface descriptions. The bonding interaction strength of the $\mathrm{O}$-donor complex was 3 to 4 times greater than that of the S-donor complex. It was because, in $O_{\mathrm{h}}$ symmetry, d-type AOs and p-type AOs belong to different irreducible representation, whereas, in $D_{3}$ symmetry, d-type AOs $\left(x^{2}-y^{2}, x y, x z\right.$ and $\left.y z\right)$ and p-type AOs $(x$ and $y)$ belong to same irreducible representation. It is indicated that the mixing of d-type AOs of metal ion and p-type AOs of donor atoms leads to the increase of MOOP for $\mathrm{S}$-donor complex. Interestingly, there were no differences in the bonding properties of the Eu and Am complexes in either $\mathrm{S}$ - or O-donor systems. This suggests that the analogous contribution of d-type AOs to the interaction between the metal ion and the donor atoms results in the formation of similar equilibrium structures in the Eu and Am complexes. In contrast, in the case of f-type AOs, the bonding properties were significantly different depending on the metal ion and ligands, as shown in Figure 6. In the case of the Eu complex, there was no overlap with the S-donor atoms and weakly positive overlap with the $\mathrm{O}$-donor atoms, indicating a non-bonding interaction in the S-donor complex and a bonding interaction in the O-donor complex, as observed in the MO surface descriptions. Meanwhile, the f-orbital of Am(III) ion has a positive overlap with the S-donor atoms and a strongly negative overlap with the $\mathrm{O}$-donor atoms, indicating a bonding interaction in the S-donor complex and an anti-bonding interaction in the $\mathrm{O}$-donor complex. This suggests that the bonding interaction between the 5 f-orbital in Am(III) ion and the S-donor atoms stabilized $\left[\mathrm{Am}\left(\mathrm{Me}_{2} \mathrm{PS}_{2}\right)_{3}\right]$ relative to $\left[\mathrm{Eu}\left(\mathrm{Me}_{2} \mathrm{PS}_{2}\right)_{3}\right]$. In contrast, the anti-bonding interaction between the 5f-orbital in $\mathrm{Am}(\mathrm{III})$ ion and the $\mathrm{O}$-donor atoms and the bonding interaction between the $4 \mathrm{f}$-orbital in Eu(III) ion and the O-donor atoms may result in destabilization of $\left[\mathrm{Am}\left\{\left(\mathrm{Me}_{2} \mathrm{PO}_{2}\right)_{2} \mathrm{H}\right\}_{3}\right]$ relative to $\left[\mathrm{Eu}\left\{\left(\mathrm{Me}_{2} \mathrm{PO}_{2}\right)_{2} \mathrm{H}\right\}_{3}\right]$. This difference in the f-orbital contributions to covalent interactions was a key factor in determining the $\mathrm{Am} / \mathrm{Eu}$ selectivity of $\mathrm{S}$ - and O-donor ligands, although the correlation between the orbital contribution to covalency and the relative stability of the Am/Eu system was as yet undetermined and should be discussed carefully in our future work.

\section{Conclusion}

Relativistic DFT calculations were applied to the chemical separation of Am(III) complex from Eu(III) complex with Sand $\mathrm{O}$-donor ligands to elucidate the correlation between the bonding properties and the Am/Eu selectivity. The computational stabilization energies of complexation with $\mathrm{S}$ - and $\mathrm{O}$-donor ligands reproduced the experimentally observed selectivity, where S-donor ligands selectively coordinate to $\mathrm{Am}(\mathrm{III})$ ion rather than $\mathrm{Eu}(\mathrm{III})$ ion and O-donor ligands preferably coordinate to Eu(III) ion rather than Am(III) ion regardless of the conformers of their complexes. Mulliken's atomic spin population analysis revealed that the $\mathrm{d}$ - and f-orbital electrons in the metal ions majorly contribute to the chemical bonding. MOOP analysis implied that the contributions of d-orbital electrons are almost same for $\mathrm{Eu}(\mathrm{III})$ and Am(III) complexes, whereas the contributions of the f-orbital electrons are significantly different. The similar interactions of the d-orbital electrons in both the Eu and Am complexes are suggested to contribute to the analogous geometries of the Eu and Am complexes. The difference in the contributions of the f-orbital electrons of Eu and Am ions is implied to be the ori- gin of the Am/Eu selectivity. It is interesting to note that both the d- and f-orbital electrons in the metal ions play important roles in formation of covalent bonding; however, their functions are different. We hope that the present work will be useful to elucidate the separation behavior of MAs from lanthanides and will contribute to the improvement and optimization of partitioning and transmutation technologies.

\section{References}

(1) H. Oigawa, K. Nishihara, S. Nakayama, Y. Morita, Proceedings of Actinide and Fission Product Partitioning and Transmutation $10^{\text {th }}$ Information Exchange Meeting (OECD/NEA, San Francisco, California, USA, 2012), p.123.

(2) K. L. Nash, Solvent Extr. Ion Exch. 11, 729 (1993).

(3) Z. Kolarik, Chem. Rev. 108, 4208 (2008)

(4) S. Tachimori, Y. Morita, Ion Exchange and Solvent Extraction: A Series of Advances, Volume 19, edited by B. A. Moyer (Routledge, 2009).

(5) S. A. Ansari, P. N. Pathak, P. K. Mohapatra, V. K. Manchanda, Chem. Rev. 112, 1751 (2012).

(6) M. P. Jensen, A. H. Bond, Radiochim. Acta 90, 205 (2002).

(7) Y. Zhu, Y. Chen, R. Jiao, Solvent Extr. Ion Exch. 14, 61 (1996).

(8) M. Watanabe, R. Mirvaliev, S. Tachimori, K. Takeshita, Y. Nakano, K. Morikawa, R. Mori, Chem. Lett. 12, 1230 (2002).

(9) R. G. Pearson, Chemical Hardness (Wiley-VCH Verlag $\mathrm{GmbH}$, Weinheim, 1997).

(10) Y. Nagame, M. Hirata, H. Nakahara, Handbook of Nuclear Chemistry, Vol. 2, $2^{\text {nd }}$ edition, edited by A. Vértes, S. Nagy, Z. Klencsár, R. G. Lovas, F. Rösch (Springer Verlag, 2011).

(11) N. Kaltsoyannis, Chem. Soc. Rev. 32, 9 (2003).

(12) G. Schreckenbach, G. A. Shamov, Acc. Chem. Rev. 43, 19 (2010).

(13) C. Platas-Iglesias, A. Roca-Sabio, M. Regueiro-Figueroa, D. Esteban-Gomez, A. de Blas, T. Rodriguez-Blas, Curr. Inorg. Chem. 1, 91 (2011).

(14) D. Wang, W. van Gunsteren, Z. Chai, Chem. Soc. Rev. 41, 5836 (2012).

(15) J. Lan, W. Shi, L. Yuan, J. Li, Y. Zhao, Z. Chai, Coord. Chem. Rev. 256, 1406 (2012).

(16)X. Cao, A. Weigand, Computational Methods in Lanthanide and Actinide Chemistry, edited by M. Dolg (John Wiley \& Sons, Ltd., 2015).

(17) X. Cao, D. Heidelberg, J. Ciupka, M. Dolg, Inorg. Chem. 49, 10307 (2010)

(18) X. Cao, J. Zhang, D. Weissmann, M. Dolg, X. Chen, Phys. Chem. Chem. Phys. 17, 20605 (2015).

(19)D. A. Pantazis, X. Chen, C. R. Landis, J. Chem. Theory Comput. 4, 908 (2008).

(20) D. A. Pantazis, F. Neese, J. Chem. Theory Comput. 5, 2229 (2009).

(21)D. A. Pantazis, F. Neese, J. Chem. Theory Comput. 7, 677 (2011).

(22) M. Kaneko, S. Miyashita, S. Nakashima, Inorg. Chem. 54, 7103 (2015).

(23) M. P. Jensen, A. H. Bond, J. Am. Chem. Soc. 124, 9870 (2002).

(24) S. Chatterjee, E. N. Maslen, K. Watson, J. Acta Crystallogr. Sect. B 44, 381 (1996).

(25) P. Lindquist-Reis, C. Apostolidis, J. Rebizant, A. Morgenstern, R. Klenze, O. Walter, T. Fanghänel, R. G. Haire, Angw. Chem. Int. Ed. 46, 919 (2007).

(26) Y. Meseri, A. A. Pinkerton, G. Chapuis, J. Chem. Soc. Dalton Trans., 725 (1977).

(27) G. Tian, Y. Zhu, J. Xu, Solvent Extr. Ion Exch. 19, 993 
(2001).

(28) E. A. Mowafy, H. F. Aly, Solvent Extr. Ion Exch. 20, 177 (2002).

(29) R. Carr, N. H. Evans, D. Parker, Chem. Soc. Rev. 41, 7673 (2012).

(30) K. Ishimori, M. Watanabe, T. Kimura, T. Yaita, T. Yamada, Y. Kataoka, S. Shinoda, H. Tsukube, Chem. Lett. 34, 1112 (2005).

(31)F. Neese, WIREs Comput. Mol. Sci. 2, 73 (2012).

(32) E. van Lenthe, E. J. Baerends, J. G. Snijders, J. Chem. Phys. 89, 4597 (1993).

(33) C. van Wüllen, J. Chem. Phys. 109, 392 (1998).

(34)K. G. Dyall, K. Fægri Jr., Introduction to Relativistic Quantum Chemistry, edited by K. G. Dyall, K. Fægri Jr. (Oxford University Press, 2007).

(35) A. D. Becke, Phys. Rev. A 38, 3098 (1989).
(36) J. P. Perdew, Phys. Rev. B 33, 8822 (1986).

(37) S. Grimme, J. Chem. Phys. 124, 034108 (2006).

(38) F. Neese, J. Comput. Chem. 24, 1740 (2003).

(39)F. Neese, F. Wennmohs, A. Hansen, U. Becker, Chem. Phys. 356, 98 (2009).

(40) K. Momma, F. Izumi, J. Appl. Crystallogr. 41, 653 (2008).

(41) R. S. Mulliken, J. Chem. Phys. 23, 1833 (1955).

(42) R. S. Mulliken, J. Chem. Phys. 23, 2338 (1955).

(43) M. P. Jensen, R. Chiarizia, I. A. Shkrob, J. S. Ulicki, B. D. Splindler, D. J. Murphy, M. Hossain, A. Roca-Sabio, C. Platas-Iglesias, A. de Blas, T. Rodríguez-Blas, Inorg. Chem. 53, 6003 (2014).

(44) M. Kaneko, S. Miyashita, S. Nakashima, Croat. Chem. Acta 88, 347 (2016).

(45) M. Kaneko, M. Watanabe, T. Matsumura, Dalton Trans. 45, 17530 (2016). 